Escritos Contables y de Administración, ISSN 1853-2063, vol. 6, n. 1, 2015, págs. 15 a 43.

\title{
EVOLUCIÓN DE LAS FUNCIONES DE UTILIDAD PARA LA TOMA DE DECISIONES
}

\section{THE UTILITY FUNCTION EVOLUTION FOR DECISION \\ MAKING}

Emilio El Alabi, Gastón Milanesi*

Fecha de recepción: 18/12/2015

Fecha de aceptación: 10/03/2016

\section{Resumen}

La toma de decisiones bajo riesgo e incertidumbre ha sido estudiada desde hace cientos de años con el objetivo de establecer un consenso normativo en el verdadero comportamiento de las personas ante este tipo de situaciones. En el siguiente trabajo, se presenta una reseña bibliográfica desarrollando, de manera cronológica, la evolución de las funciones de utilidad postuladas por los autores más relevantes en esta rama de estudio. Si bien son numerosos los autores que han abordado el tema, se comenzará explicando la teoría del valor esperado, la paradoja de San Petersburgo, la teoría de la utilidad esperada, el aporte tanto de Friedman y Savage como de Markowitz, para culminar detallando la teoría prospectiva de Kahneman y Tversky.

Palabras clave: toma de decisiones, funciones de utilidad, riesgo e incertidumbre.

\footnotetext{
* Universidad Nacional del Sur, Departamento de Ciencias de la Administración.

E-mail: emilio.elalabi@uns.edu.ar, milanesi@uns.edu.ar
} 
Emilio El Alabi, Gastón Milanesi

\begin{abstract}
Academics have been studying decision making under risk and uncertainty for hundreds of years in order to determine the actual behavior of individuals. Throughout this paper, we present, chronologically, the most relevant state of the art in utility functions developed by the most well-known authors. Even though there are numerous authors who have studied the subject, we begin explaining expected value theory (Huygens, 1657), St. Petersburg Paradox (Bernoulli, 1738), expected utility theory (von Neumann \& Morgenstern, 1944), contributions not only from Friedman and Savage (1948) but also from Markowitz (1952), and we end up detailing prospect theory (Kahneman \& Tversky, 1979).
\end{abstract}

Keywords: decision making, utility functions, risk and uncertainty.

JEL: D01, D03, D81. 


\section{Introducción}

Desde Huygens (1657) pasando por Kahneman y Tversky (1979) hasta la actualidad, la toma de decisiones en condiciones de incertidumbre ha sido foco de atención de innumerables investigaciones dada la imperativa necesidad de establecer un marco normativo que postule el comportamiento de los agentes frente al riesgo. Los primeros modelos partieron con la concepción de un ser humano simétrico frente al riesgo en cuanto a aumentos o disminuciones de su riqueza, cuyo principal postulado consistía en la acumulación de riqueza. Su conducta frente al riesgo es explicada a través de un conjunto de axiomas que sirven de perímetro o marco referencial a la toma de decisiones. Estos modelos evolucionaron hacia un agente asimétrico en relación con sus actitudes frente al riesgo, ahora en el caso de pérdidas y ganancias, en lugar de riqueza acumulada. Fueron adquiriendo complejidad en búsqueda de explicar comportamientos que se aproximaran mejor a las observaciones capturadas en los experimento de laboratorios, sin perder de vista el objetivo de maximizar la utilidad esperada del agente. Es así que el concepto de probabilidad objetiva cede frente a las probabilidades subjetivas, la condición o estrato social explica en parte la actitud frente al riesgo, y el tradicional concepto de racionalidad vira hacia un ser limitadamente racional. En el mismo, los puntos de referencia y sesgos en las ponderaciones de probabilidades vinculadas a pérdidas o ganancias definen un nuevo formato de función de utilidad. No se debe perder de vista que, durante este recorrido, también es concebido el instrumento para maximizar la utilidad esperada del individuo, conocido como criterio media-varianza.

El trabajo resume los principales conceptos correspondientes a las diferentes formulaciones teóricas para explicar el comportamiento de los individuos frente a la toma de decisiones en condiciones de incertidumbre. Por tal motivo, pretende ser un documento de consulta inicial para todos aquellos interesados en profundizar sobre los diferentes modelos que prescriben el comportamiento humano frente al riesgo. El tema es de vital importancia, en particular desde la perspectiva de la teoría financiera, entre 
otros campos del conocimiento. A modo de ejemplo, el concepto tradicional de prima por riesgo ${ }^{1}$ siempre estudiado desde la perspectiva de los precios de mercado, reconoce su génesis y explicación en el supuesto de aversión al riesgo de los agentes, explicado por la teoría de la utilidad esperada. Conforme fue indicado, el concepto de maximización de la utilidad esperada tiene su faz operativa en el criterio media-varianza y la asignación de recursos en carteras de activos eficientes frente a un conjunto de oportunidades de inversión establece los postulados de la teoría moderna de la cartera.

La estructura del trabajo es la siguiente: en la siguiente sección, se analiza la evolución y críticas a la teoría de la utilidad esperada desde los iniciales conceptos de Huygens (1657), pasando por la formulación de Bernoulli (1738), hacia los axiomas de la teoría de la utilidad planteados por Von Neumann y Morgenstern (1944). Seguidamente, se presentan las curvas de utilidad de Friedman y Savage (1948) y Markowitz (1952). Respecto de este último, se explica la relación entre el criterio de elección media varianza y la maximización de la utilidad esperada. Luego, se introducen las críticas de Allais (1953, 1988), Savage (1954) y Simon (1955, 1975, 1978, 1979, 1986). La tercer sección explican los supuesto, los postulados y la forma de la función de valor propuesta por Kahneman y Tversky (1979) como nuevo paradigma en la formulación de la conducta del individuo frente al riesgo. Finalmente, se exponen las principales conclusiones.

\section{Evolución y críticas a la teoría de la utilidad esperada}

\subsection{Teoría de la utilidad}

El conocimiento de la toma de decisiones en condiciones de incertidumbre es objeto de estudio desde el siglo XVII. Uno de los pioneros en la materia fue Huygens (1657) quien introduce el concepto de valor esperado de la decisión. Comienza analizando un juego de azar simétrico, donde los eventos $a$ y $b$ tienen igual probabilidad de ocurrencia. Consecuentemente, el valor esperado de la apuesta está dado por

$$
(a+b) / 2 \quad(1)
$$


Huygens toma como ejemplo el caso donde un sujeto es parte de un juego donde tiene igual oportunidad de ganar $\$ 3$ o ganar $\$ 7$, siendo el valor de la expectativa de $\$ 5$. Sostiene que esta situación es equivalente a un juego donde participan dos jugadores, con un costo de entrada de $\$ 5$. El jugador que resulte vencedor se llevará los \$10 apostados pero deberá pagarle al perdedor $\$ 3$; es decir el perdedor obtiene $\$ 0$ pero le otorgan $\$ 3$. El ganador obtiene $\$ 10$ pero otorga $\$ 3$ al perdedor. El resultado final está dado porque el perdedor y el ganador del juego finalizan con $\$ 3$ y $\$ 7$, respectivamente. Posteriormente, Huygens plantea el concepto de valor esperado en situaciones donde las probabilidades de ocurrencia correspondientes a los eventos $a$ o $b$ no son simétricas. Para ello, supone que las probabilidades asociadas a los eventos están dadas por $p$ y $q$, siendo el valor esperado de la decisión explicado por la siguiente ecuación:

$$
(a p+b q) /(p+q)
$$

De la expresión anterior se deriva la ecuación correspondiente al valor esperado aplicable a decisiones en condiciones de incertidumbre con $n$ probabilidades de ocurrencia. La expresión es

$$
\sum_{i=1}^{n}\left(a_{i} * p_{i} / \sum_{i=1}^{n}\left(p_{i}\right)\right)
$$

donde $a_{i}$ representa el $i$-ésimo resultado posible y $p_{i}$ la posibilidad de obtener $a_{i}$, siendo este planteo una aproximación del valor esperado correspondiente a un juego riesgoso.

Posteriormente, Daniel Bernoulli (Dehling, 1997) presenta las primeras críticas a la teoría del valor esperado como medida en el proceso decisorio. Conocida como paradoja de San Petersburgo presenta un juego en donde un apostador arrojando una moneda puede obtener dos posibles resultados "cara-cruz". El sujeto tira la moneda hasta obtener por primera vez como resultado el lado conocido como "cara", perdiendo el juego. La recompensa del juego planteado por Bernoulli consistía en un pago de 2 florines en el caso de que el resultado no deseado se obtenga en la primera tirada, 4 florines si ocurre en la segunda tirada, 8 en la tercera, 16 si ocurre en la cuarta, y así en adelante. La probabilidad de obtener el resultado 
"cara" en la primera tirada es de $1 / 2$, en la segunda de $1 / 4$, en la tercera de $1 / 8$, en la cuarta de $1 / 16$ y así sucesivamente.

Esta situación conduce al siguiente interrogante: ¿cuánto estaría dispuesto a pagar un hombre razonable por aceptar ingresar en el juego? Hasta ese momento, la respuesta era dada por la teoría del valor esperado (ecuación 3). En este caso, el valor del juego es igual a

$$
2 * \frac{1}{2}+4 * \frac{1}{4}+8 * \frac{1}{8}+16 * \frac{1}{16}+\cdots=\infty \ldots \text { infinito! }
$$

Por otro lado, se podría plantear la pregunta si alguien estaría dispuesto a pagar 100 florines por participar en el juego. Matemáticamente, para recuperar los 100 florines, debería salir "cruz" en las primeras 6 tiradas del juego, este evento tiene una probabilidad de ocurrencia de 1/64. Las chances de perder algo de riqueza en el juego son de 63 a 1 y, en principio, se puede considerar como una situación bastante riesgosa para el común de los agentes. Por lo tanto, el valor esperado de este juego es infinito (ecuación 3) pero no parece razonable pagar, por ejemplo, una suma de 100 florines si las chances de ganar son extremadamente bajas.

Mediante este sencillo ejemplo, Bernoulli introduce la noción de utilidad, ya que analiza la situación de una persona que recibe un billete de lotería con igual chance de ganar 20.000 ducados o nada. El autor sostiene que un hombre pobre podría venderlo por 9.000 ducados y un hombre rico estaría tentado a comprarlo por 9.000 ducados. Para Bernoulli, aplicar la regla del valor esperado para evaluar la situación puede conducir a conclusiones incorrectas. Por ello, propone analizar en juego no en términos de precio (valor esperado), sino plantearlo en términos de utilidad. En este caso, el concepto difiere de la tradicional concepción de precio, ya que este es un fenómeno observable similar para todos los agentes. A diferencia de la utilidad, este concepto es subjetivo e individual condicionado al estado de riqueza en que se encuentra el individuo ${ }^{2}$.

Este ejemplo sirvió como base para justificar la forma funcional correspondiente a la primera función de utilidad. Es decir, una curva de utilidad de forma cóncava, donde la utilidad que resulte de cualquier pequeño incremento en la riqueza de un individuo va a ser inversamente 
proporcional a la cantidad total de bienes que ese individuo poseía previamente (Bernoulli, 1738) 3 .

Sobre el trabajo de Bernoulli, Von Neumann y Morgenstern (1944) desarrollan la teoría de la utilidad esperada como sostén teórico para fundamentar un mecanismo racional de toma de decisiones en condiciones de incertidumbre, explicando la conducta de los agentes con el objetivo de llegar sistemáticamente al óptimo de la decisión. Para esto, los autores proponen cuatro axiomas de comportamiento, ya que si el orden de preferencias de los agentes se ajusta a dichos axiomas, entonces el resultado de dicha conducta conduce a una maximización de la utilidad. Para la definición de los axiomas, se asume que el individuo tiene preferencias definidas sobre un conjunto potencial de loterías. Los axiomas que proponen son los siguientes:

a. Completitud. Dadas dos loterías posibles, el individuo tiene la posibilidad de definir si prefiere una $u$ otra, o si es indiferente entre las dos.

b. Transitividad. Imagine tres loterías $L_{1}, L_{2}$ y $L_{3}$. Si el individuo prefiere $L_{1}$ a $L_{2}$ y $L_{2}$ a $L_{3}$, entonces el individuo debe preferir $L_{1}$ a $L_{3}$. Es decir, $L_{1}>L_{2}$ y $L_{2}>L_{3}$, entonces $L_{1}>L_{3}$.

c. Continuidad. El individuo debe ser capaz de asignar una probabilidad $p$ de modo que le sea indiferente elegir una lotería intermedia simple $L_{2}$ o una lotería compuesta $p L_{1}+(1-p) L_{3}$.

d. Independencia. La noción de este axioma hace referencia a que dadas dos loterías $L_{1}$ y $L_{2}$, las preferencias entre ellas no se ve afectada si se incluye una tercera lotería $L_{3}$.

De aquí surgen los conceptos de valor esperado de la riqueza, $E(x), \mathrm{y}$ utilidad esperada , $E[U(x)]$, siendo

$$
E(x)=\sum_{i=1}^{n} p_{i} \times x_{i}(4)
$$

donde $p_{i}$ representa la probabilidad de ocurrencia asociada al i-ésimo evento y $x_{i}$ la riqueza correspondiente a dicho estado de la naturaleza.

$$
E[U(x)]=\sum_{i=1}^{n} p_{i} \times u\left(x_{i}\right)
$$


donde $p_{i}$ representa la probabilidad de ocurrencia asociada al i-ésimo evento; $x_{i}$ la riqueza correspondiente a dicho estado de la naturaleza y $u($. la función de utilidad para la riqueza.

La forma de la función de utilidad $u($.$) se explica en la figura 1. Allí, se$ puede observar que las personas tienen en cuenta su riqueza total para determinar el grado de utilidad que les representa una decisión. A diferencia de Bernoulli, consideran dos clases de individuos ${ }^{4}$. Una persona que es adversa al riesgo y otra que es afecta al riesgo. Las consecuencias económicas de la forma funcional del individuo adverso al riesgo explica una función de utilidad creciente y cóncava debido a:

a. No satisfacción. Dadas dos alternativas ciertas $x_{1}=(x+\$ 1)$ y $x_{2}=$ $(x-\$ 1)$, entonces $U_{x_{1}}>U_{x_{2}} ; x_{1} \geqslant x_{2}$, los axiomas aplicados son completitud e independencia. Esto implica que se prefiere más riqueza a menos riqueza, entonces la curva de utilidad es creciente y su primer derivada positiva $\left(U^{\prime}{ }_{x}>0\right)$.

b. Actitudes frente al riesgo. Para un agente adverso al riesgo, supóngase una lotería en donde el valor esperado de su riqueza $E(x)$ (ecuación 4) correspondiente a la opción "inversión" es $E(x)=0,5 \times \$ 2+0,5 \times 0=\$ 1$. Se supone que el inversor debe pagar en $t=0$, la suma de $\$ 1$ para en el futuro $t=1$ ganar $\$ 2$ con $50 \%$ de probabilidad o $\$ 0$ con el complemento $50 \%$. Por lo tanto, $E(x)$ se fija como costo de la inversión. Bajo dichas condiciones, un sujeto adverso al riesgo elige la opción de "no inversión" ya que $\$ 1$ seguros, suma que debe abonar en el presente, son preferidos a $\$ 1$ esperados $E(x)=0,5 \times \$ 2+0,5 \times 0=\$ 1$. Matemáticamente, la segunda derivada de la función de utilidad es negativa $\left(U^{-}{ }^{-} x\right.$ $<0)$. Esto implica que la utilidad esperada $E[U(x)]$ (ecuación 5) de "no inversión" es mayor que la de "inversión". La forma de la función de utilidad es cóncava, a partir del ejemplo $U(\$ 1)>0,5 \times$ $U(\$ 2)+0,5 \times U(0)$. Reordenando y multiplicando por 2 ambos lados: $U(\$ 1)-U(\$ 0)>U(\$ 2)-U(0)$. Esto indica que tiene un mayor nivel de utilidad un cambio de $\$ 0$ a $\$ 1$ que incrementar riqueza de $\$ 1$ a $\$ 2$. Consecuentemente, una función donde unidades adicionales añaden menor valor tiene una derivada 
segunda negativa ${ }^{5}$. En este caso, el sujeto adverso al riesgo acepta el juego si su costo es inferior a la utilidad esperada. En otras palabras, la utilidad del valor esperado (costo) debe ser menor que el valor de la utilidad esperada; $U[E(x)]<E[U(x)]$. En el caso de un sujeto afecto al riesgo, la relación es inversa; $U[E(x)]>$ $E[U(x)]$.

Figura 1. Función de utilidad de von Neumann y Morgenstern (1944)

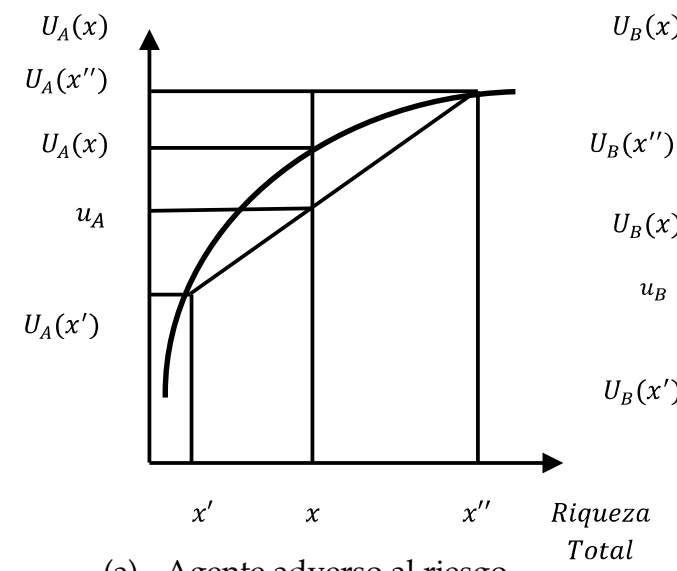

(a) Agente adverso al riesgo

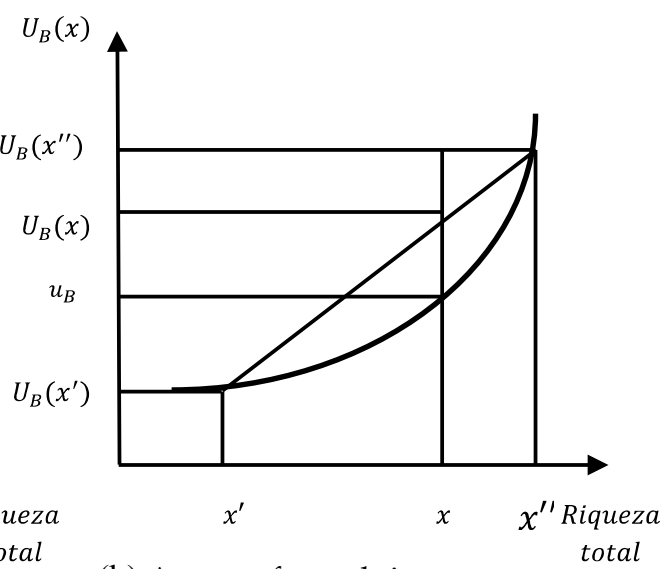

(b) Agente afecto al riesgo

Fuente: elaboración propia 6 .

Un detalle importante a considerar con respecto a la teoría de la utilidad esperada es que se asume que las personas son siempre adversas o siempre afectas al riesgo. Es decir, no cambian sus preferencias ante el riesgo. Por lo tanto, no sería consistente que la misma persona contrate un seguro (demostrando adversidad ante el riesgo) y compre un billete de lotería (demostrando afecto al riesgo) (Chaz Sardi, 2015).

\subsection{Nuevas funciones de utilidad}

Cuatro años más tarde, Friedman y Savage (1948) proponen una nueva curva de utilidad. A diferencia de la curva de utilidad propuesta por Von Neumann y Morgenstern, no considera la riqueza total como la base para determinar el nivel de utilidad. En esta, la variable está dada por el nivel 
de ingreso del individuo. La nueva curva de utilidad contempla 5 atributos del comportamiento de los individuos ante el riesgo: a) los consumidores prefieren mayores ingresos seguros que menores ingresos seguros; b) los individuos de bajos ingresos compran, o están dispuestos a comprar, seguros; c) las personas de bajos ingresos compran, o están dispuestos a comprar, billetes de lotería; d) muchos de estos sujetos compran, o están dispuestos a comprar, tanto seguros como billetes de lotería y e) por lo general estas loterías tienen más de un precio.

Figura 2. Curva de utilidad de Friedman y Savage (1948)

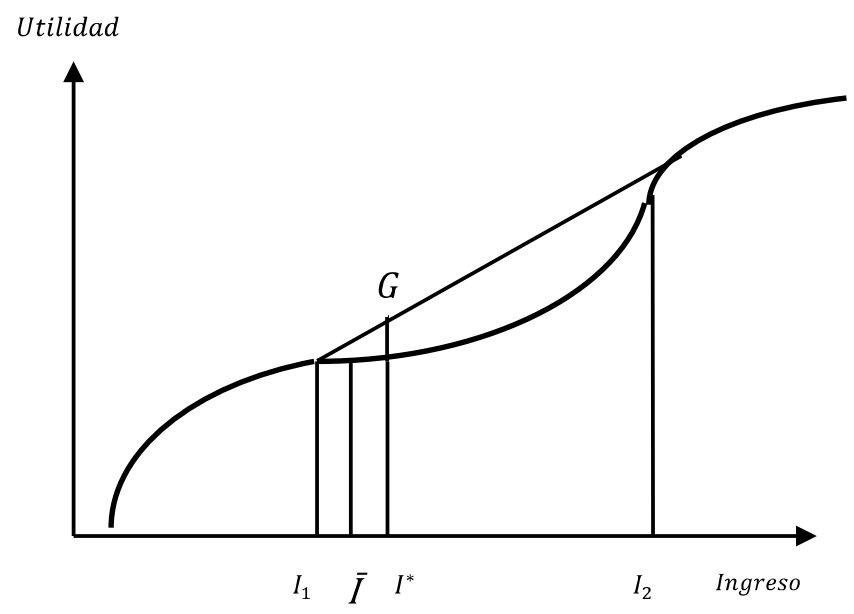

Fuente: elaboración propia.

Para ilustrar la lógica de esta nueva función de utilidad y sus atributos empleados para describir la conducta de los individuos, imagine un trabajador no calificado que tiene un ingreso bajo. Su salario actual se encuentra en la posición $I^{*}$ de la figura 2 . Suponga que se presenta cierto riesgo de una pérdida, el individuo estaría dispuesto a comprar un seguro contra ese riesgo (siempre que no tenga que pagar demasiado) ya que, en el caso de sufrir esta pérdida, su ingreso estaría determinado entre el comienzo de la curva e $I_{1}$. Este individuo no estaría dispuesto a jugar apuestas de bajo monto. Sin embargo, asuma que se le ofrece jugar una apuesta justa ${ }^{7}$ donde tiene una pequeña probabilidad de ganar un importe significativo igual a $I_{2}-I^{*}$ y una gran probabilidad de perder poco, es decir una suma igual a $I^{*}-I_{1}$. 
Esta persona claramente aceptaría la apuesta, ya que la utilidad esperada $E[U(G)]=p_{a} \times\left(I_{2}-I^{*}\right)+p_{b} \times\left(I^{*}-I_{1}\right) ; \quad$ siendo $p_{a}<p_{b}$ es mayor a la utilidad de $I^{*}$. Por lo tanto, $U(I)<E[U(G)]$, conforme se puede apreciar en el gráfico. De hecho, estaría dispuesto a pagar $I^{*}-\bar{I}$ por el privilegio de entrar en la apuesta. Incluso si el valor esperado de la apuesta es igual a $\bar{I}$, el individuo aceptaría la apuesta por encima de aceptar $I^{*}$ de manera segura. De esta manera, esta curva de utilidad es consistente con los atributos b), c) y d) presentados anteriormente.

Cabe recordar que este comportamiento se refiere únicamente a trabajadores de bajo ingreso. Resulta muy tentador restringir aún más los supuestos para poder abordar el comportamiento de trabajadores de ingresos mayores. De todas maneras, los autores no extienden la explicación a sujetos de ingresos mayores por dos razones principales: por un lado, es mucho más complejo obtener información de algunos individuos de ingresos altos que de muchos más individuos de ingresos bajos. Por otro lado, y tal vez más importante, el impuesto progresivo aplicado a los ingresos afecta la manera en que los trabajadores de ingresos altos compran seguros o incursionan en una lotería (Friedman y Savage, 1948).

En su trabajo, los autores se preguntan sobre el realismo de la hipótesis planteada. Es decir, indagan sobre si los individuos efectivamente consultan una curva de utilidad con concavidades y convexidades alternadas ante la decisión de comprar un seguro o apostar su dinero si conocen las probabilidades asociadas a un seguro o esa apuesta y si basan sus decisiones solamente en la magnitud de la utilidad esperada. Concluyen que, al margen de si las personas deciden de manera analítica o lo hacen naturalmente, el modo en que lo hacen es irrelevante ya que los individuos se comportan como si hubiesen aplicado el modelo mental descripto para la toma de decisiones. Entonces, el modelo propuesto sirve de marco formal para explicar la conducta de los agentes frente a elecciones en condiciones de incertidumbre.

La explicación presentada por Friedman y Savage en relación con la curva de utilidad desarrollada y el comportamiento de los individuos según su nivel de riqueza es la siguiente: a) las dos partes cóncavas de la curva corresponden a distintos estratos socioeconómicos, b) la parte convexa 
corresponde a la transición entre los dos estratos. La intuición que subyace en la figura 2 es la siguiente: a) un incremento en el ingreso del trabajador que no lo catapulta a una nueva clase socioeconómica tiene una utilidad marginal decreciente; b) un incremento en el ingreso que promueva a ese mismo trabajador a una nueva clase tiene una utilidad marginal creciente. Bajo este razonamiento, un trabajador no calificado no aceptará una apuesta justa donde, en el caso de ganar, lo coloque entre los mejores trabajadores no calificados y, en el caso de perder, lo coloque entre los peores trabajadores no calificados. Sin embargo, él aceptará una apuesta justa que ofrezca una pequeña posibilidad de saltar de la clase socioeconómica donde se encuentra, incluso si la posibilidad es más grande que el resultado de la apuesta lo coloque entre los peores trabajadores no calificados. Los hombres toman y tomarán grandes riesgos con el objeto de sobresalir, aun sabiendo cuáles son los riesgos a los que se están enfrentando.

En su trabajo Markowitz (1952) presenta observaciones a la curva de utilidad propuesta por Friedman y Savage, y propone una nueva curva de utilidad. Critica ciertos comportamientos descriptos por la curva de utilidad de Friedman y Savage. La particularidad de este enfoque consiste en que determina la utilidad de la decisión a partir del valor de la decisión pero no enfocado en la riqueza total o el nivel de ingreso acumulado del agente ${ }^{8}$. Consecuentemente, divide la curva de Friedman y Savage en tres partes para describir tres tipos de comportamientos.

Figura 3. Curva de utilidad de Friedman y Savage presentada por Markowitz (1952)

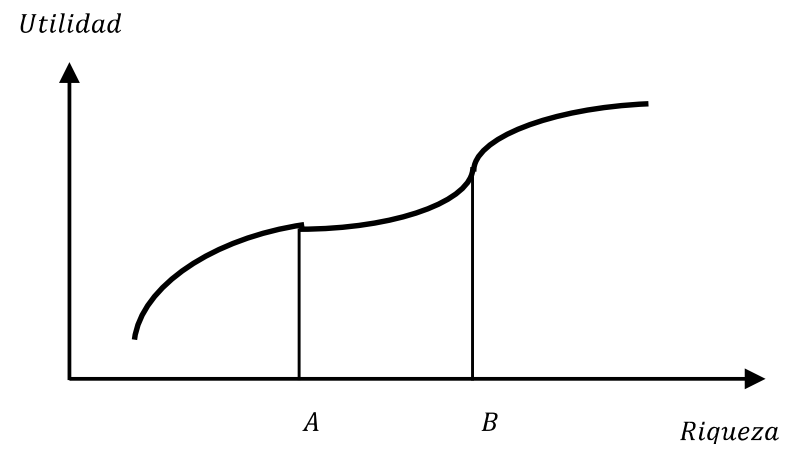

Fuente: elaboración propia. 
La interpretación de la curva es la siguiente: supóngase un sujeto que se encuentra con una riqueza justo en el punto medio entre $A$ y $B$; es decir $((A+B) / 2)$ de la figura 3. De acuerdo con Friedman y Savage, esa persona estaría dispuesta a incursionar en una apuesta justa en la que tire una moneda y si sale "cara", su riqueza será igual a $B$, y si sale "cruz", su riqueza será igual a $A$; es un juego más que tentador para el sujeto, situación no observada empíricamente en sus estudios por Markowitz. Seguidamente, considere una persona que tiene una riqueza apenas por debajo de $B$, en este caso un sujeto con una dotación significativa. De acuerdo con Friedman y Savage, la apuesta más tentadora para este sujeto es aquella en la que, si gana lo catapulte por encima de $B$, pero si pierde, lo sepulte por debajo de A, pero solamente estaría dispuesto a tomarla si las probabilidades de obtener una ganancia pequeña son grandes y las probabilidades de sufrir una gran pérdida son pequeñas. En este caso, no se aseguraría para el caso de sufrir una pérdida. De hecho, estaría dispuesto a extender un seguro contra su propia pérdida. Nuevamente, Markowitz no encontró evidencia empírica de esta predicción.

Este autor propone indagar sobre el comportamiento común frente al riesgo de sujetos con menos riqueza que $A$ o más riqueza que $B$, estableciendo que nunca incursionarían en una apuesta justa, independientemente del nivel de ingresos. Tanto personas ricas como pobres suelen comprar tickets de lotería, apostar en los caballos, o participar en cualquier otro tipo de apuestas, sin distinción de su nivel de ingresos o riqueza, jugando por placer o por disponer de información asimétrica. El autor desarrolla su propia curva de utilidad utilizando el método científico de laboratorio sobre una muestra de agentes controlada. Para ello, comienza realizando preguntas hipotéticas de manera informal. En primer lugar, se formula la pregunta en términos de ganancias y a medida que los sujetos son interrogados se procede a incrementar los montos. Seguidamente, la pregunta se realiza en término de pérdidas. En total, son 11 las preguntas del cuestionario con el fin de indagar sobre las preferencias de los agentes. Las preguntas son:

1. Ganar 10 centavos de manera segura o una chance en 10 en ganar $\$ 1$.

2. Ganar $\$ 1$ de manera segura o una chance en 10 de ganar $\$ 10$. 
3. Ganar $\$ 10$ de manera segura o una chance en 10 de ganar $\$ 100$.

4. Ganar $\$ 100$ de manera segura o una chance en 10 de ganar $\$ 1.000$.

5. Ganar $\$ 1.000$ de manera segura o una chance en 10 de ganar $\$ 10.000$.

6. Ganar $\$ 1.000 .000$ de manera segura o una chance en 10 de ganar $\$ 10.000 .000$.

7. Deber 10 centavos o una chance en 10 de deber $\$ 1$.

8. Deber $\$ 1$ o una chance en 10 de deber $\$ 10$.

9. Deber $\$ 10$ o una chance en 10 de deber $\$ 100$.

10.Deber $\$ 100$ o una chance en 10 de deber $\$ 1.000$.

11.Deber $\$ 1.000 .000$ o una chance en 10 de deber $\$ 10.000 .000$.

Según el autor, estas serían las típicas respuestas que dan forma a su curva de utilidad:

a. La mayoría prefiere tomar la oportunidad de $\$ 1$ en lugar de aceptar 10 centavos de manera segura.

b. Tomar la oportunidad de \$10 en lugar de aceptar \$1 de manera segura.

c. La situación comienza a ser dispar cuando se tiene que decidir entre la oportunidad de $\$ 100$ o aceptar $\$ 10$ de manera segura.

d. Los que aceptan los \$10, también aceptan los \$100.

e. Mientras que la mayoría que toma riesgo en la pregunta 3, también elige los \$100 de manera segura en la pregunta 4 .

f. Al llegar a la pregunta 6, todos prefieren lo seguro antes que tomar riesgo.

Con respecto a las pérdidas, las respuestas relevadas son:

a. La gente generalmente indica una preferencia a deber 10 centavos de manera segura antes que tomar la oportunidad de deber $\$ 1$.

b. Prefiere deber $\$ 1$ de manera segura antes que tomar la oportunidad de deber $\$ 10$; incluso prefiere deber $\$ 10$ que tener una chance en 10 de deber $\$ 100$.

c. Sin embargo, se llega a un punto donde el individuo prefiere la opción riesgosa. Por ejemplo, en la pregunta 11, las personas prefieren tomar riesgo y tener una chance en $\$ 10.000 .000$ que deber $\$ 1.000 .000$ de manera segura. 
La curva de utilidad de Markowitz determina que los individuos son afectos al riesgo cuando las ganancias son de bajo monto, pero que cambian sus preferencias frente al riesgo cuando los montos son más altos, y se vuelven adversos al riesgo. Con respecto a las pérdidas, las personas son adversas al riesgo en los montos más bajos y luego cambian sus preferencias cuando los montos son más altos tornándose en afectos al riesgo.

Esta curva hace referencia, al igual que la presentada por Friedman y Savage, a un individuo de bajos recursos. El autor, entonces, se pregunta cómo incide el estatus económico del individuo en la decisión. Cree que, en el caso de las ganancias, las personas tardan más en cambiar de conducta frente al riesgo. En base a su escala monetaria de la figura 4, si una persona de bajos recursos pasa de la condición afecto a adverso al riesgo en una suma de \$100; entonces una persona con un gran poder adquisitivo lo hará alrededor de los $\$ 1.000$ o, inclusive, alrededor de los $\$ 10.000$. Por lo tanto, el tercer punto de inflexión de la curva se desplaza hacia la derecha. Del mismo modo para las pérdidas, el autor intuye que las personas ricas tardan más en cambiar de conducta frente al riesgo. Gráficamente, hace que el primer punto de inflexión se desplace hacia izquierda.

Asimismo, define que las personas evitan las apuestas justas. Por lo tanto, la curva cae más rápido hacia la izquierda del punto de referencia que hacia la derecha del mismo. Para evitar la mencionada paradoja de San Petersburgo, es decir que los valores tiendan a infinito, se asume que la función es limitada en el extremo superior e inferior.

Figura 4. Curva de utilidad de Markowitz (1952)

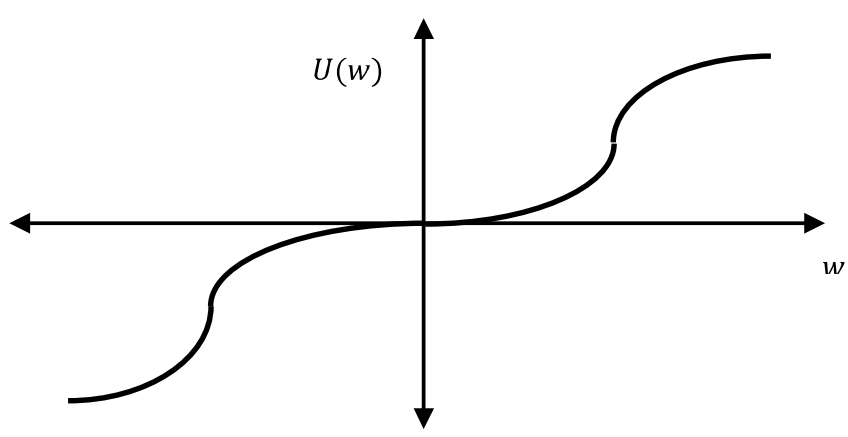

Fuente: elaboración propia. 
Uno de los principales aportes de Markowitz consistió en desarrollar el criterio de media-varianza para aproximar el concepto de maximización de la utilidad esperada $E[U(x)]$, a través de los precios observados de los activos (Markowitz, 1952; Markowitz, 1991; Markowitz, 2014). Este criterio, y su posterior evolución (Markowitz, 1959; Levy y Markowitz, 1979; Simaan, 1993; Ederington, 1996; Markowitz, 2014), es utilizado como modelo operativo para la asignación de recursos en activos riesgosos siendo la piedra basal de la teoría moderna de la cartera. El criterio se justifica a partir de distinguir tres tipos de maximización de la utilidad esperada:

a. Explícita.

b. Aproximación a través del criterio de media-varianza.

c. Implícita.

El primer caso se presenta cuando se explicita una función de utilidad y a partir de métodos analíticos se encuentra la cartera de activos que maximiza el valor esperado de la función. El segundo criterio consiste en lo siguiente: dada una función de utilidad, y tomando rendimientos y varianzas de los activos que integran la cartera, se genera una frontera eficiente de inversión, producto de combinaciones óptimas de activos que maximizan rendimiento. Por ejemplo, $E[\ln (1+r)]^{9}$, a un determinado nivel de riesgo, en los términos de la teoría de la cartera (Markowitz, 1952). Una vez generado el conjunto eficiente de posibilidades de inversión, se elige la cartera que maximiza la función de utilidad $U(r)$, y esta puede ser aproximada mediante una función cuadrática $f(r, v)$ que combina rendimiento esperado y varianza de la cartera (Markowitz, 1959). Supóngase una función cuadrática $Q_{z}(r)=U(0)+U^{\prime}(0) r+0.5^{\prime \prime}(0) r^{2}$, para la función logarítmica de utilidad $U=\ln (1+r)$, la aproximación es $Q_{z}(r)=r-1 / 2 r^{2}$ y sus valores esperados son funciones de la media $\mathrm{y}$ varianza; $f_{z}(r, v)=r-\left(r^{2}+v\right) / 2$ (Markowitz H. , 2014). Finalmente la función implícita deriva del segundo criterio, ya que aplicando el criterio media-varianza, independientemente de que el sujeto no conozca su función de utilidad, la cartera seleccionada implícita maximizará la función ya que es la combinación de rendimiento-riesgo más eficiente. (Levy y Markowitz, 1979). 
Markowitz justifica el empleo del criterio de media-varianza como aproximación a la maximización de la utilidad esperada, por cuestiones de practicidad. En el caso del criterio media-varianza, solamente se necesitan como insumos la matriz de rendimientos, varianzas y covarianzas para seleccionar la cartera eficiente que maximiza la utilidad esperada; independientemente de la forma de distribución de probabilidad, ya sea que estén normalmente distribuidos o presenten asimetría o curtosis: a) el rendimiento de la cartera siempre es $r_{p}=\sum_{i=1}^{n} x_{i} r_{i}$; siendo $x_{i} r_{i}$ la proporción invertida y el rendimiento del $i$-ésimo activo; b) la varianza de la cartera es $v_{p}=\sum_{i=1}^{n} \sum_{j=1}^{n} x_{i} x_{j} \sigma_{i, j}$; siendo $\sigma_{i, j}$ la covarianza entre el $i$-ésimo y jésimo activo. En el caso de maximizar una función explícita de utilidad, se debe estimar que tipo de distribución de probabilidad genera la combinación de rendimientos $\left(r_{1}, r_{2}, \ldots r_{n}\right)$ que maximiza la utilidad, siendo esta una tarea un tanto más compleja para arribar a similares resultados. Además, se debería indagar sobre la función de utilidad del inversor. En este caso, tal como manifestaba Von Neumann y Morgenstern (1944), se debería someter a un cuestionario a cada agente sobre el valor de la probabilidad $p_{a}$ asociada a rendimientos $r_{a}$, versus $1-p_{a}$ asociada a $r_{c}$, son consideradas equivalentes a un rendimiento cierto $r_{b}$. Esta tarea parece sencilla para un inversor pero no así para una cantidad significativa, como puede acontecer en el caso de inversores institucionales como fondos de pensión y bancos, entre otros.

\subsection{Críticas a la teoría de la utilidad esperada}

Allais (1953) desafía los axiomas de la teoría de la utilidad. A partir de preguntas hipotéticas, demuestra que los individuos violan sistemáticamente los axiomas de la utilidad esperada. En particular, no siguen el axioma de independencia. Además, demuestra que las personas no siempre buscan la maximización de la utilidad en sus decisiones. Esto fue conocido como "la paradoja de Allais". Un segundo punto a destacar dentro de esta paradoja es que los agentes pueden cambiar sus preferencias al riesgo frente a un mismo problema que se les presenta de dos maneras diferentes (Allais, 1988). 
Savage (1954) presenta una nueva crítica a la teoría de la utilidad. El autor, a diferencia de Von Neumann y Morgenstern, utiliza probabilidades personales o subjetivas dando lugar a la teoría de probabilidad subjetiva. Savage hace una distinción entre los estadísticos que utilizan las frecuencias como probabilidades objetivas de ocurrencia y una nueva corriente de estadísticos que usan las creencias de las personas como probabilidades subjetivas de ocurrencia. De acuerdo con la teoría de utilidad, todas las personas deberían tomar la misma decisión si disponen de la misma cantidad de información, ya que sus probabilidades son objetivas. Sin embargo, bajo esta nueva teoría de probabilidad subjetiva, aun teniendo la misma información, dos personas podrían tomar una decisión contraria del otro ya que sus probabilidades de ocurrencia son particulares de cada individuo.

Simon $(1955,1975,1978,1979,1986)$ presenta la idea de racionalidad limitada. En contra del postulado de la teoría de la utilidad sobre la presencia de un homo economicus y basándose en la observación en el funcionamiento de las organizaciones, desarrolla el concepto de racionalidad limitada. En ella, el agente no tiene acceso a toda la información existente $\mathrm{y}$, aunque la tuviese, no dispone de la capacidad para poder procesarla. Al momento de tomar la decisión, las personas no siguen un proceso de optimización sino que buscan la alternativa que mejor satisfaga sus necesidades personales sin realizar un planteo exhaustivo de todas las opciones posibles. A esto lo llamó satisficing model (Simon, 1979).

\section{Teoría prospectiva y las finanzas conductuales}

\subsection{Teoría prospectiva}

Kahneman y Tversky desarrollan Prospect Theory (1979), conocida como Teoría prospectiva o de perspectivas. Igual que Allais (1953), comienzan demostrando, mediante experimentos de laboratorios, las respuestas y por ende las decisiones de los agentes que integran la muestra controlada frente a situaciones hipotéticas. Se plantea como hipótesis que, en la toma de 
decisiones, las personas violan algunos de los axiomas sobre los que se basa la teoría de la utilidad esperada. Para ello, emplearon un total de 13 problemas. Los primeros 8 muestran situaciones donde las personas no se comportan de acuerdo con la teoría de la utilidad esperada. Los 5 problemas finales refuerzan las bases para construir una nueva función de utilidad. A partir de dos pares de problemas tomados del trabajo de Allais (1953), se plantea:

Problema 1, las personas tienen que decidir entre:

A. $\$ 2.500$ con una probabilidad de 0,33 .

$\$ 2.400$ con una probabilidad de 0,66

$\$ 0$ con una probabilidad de 0,01 .

B. $\$ 2.400$ de manera segura.

En este caso, la mayoría de las personas prefieren la opción B.

Problema 2, las personas tienen que decidir entre:

C. $\$ 2.500$ con una probabilidad de 0,33 . $\$ 0$ con una probabilidad de 0,67 .

D. $\$ 2.400$ con una probabilidad de 0,34 . $\$ 0$ con una probabilidad de 0,66.

En este caso, la mayoría de las personas prefieren la opción C.

Este problema viola la teoría de la utilidad esperada en la misma manera que fue explicado por Allais (1953). En el problema 2, se llegan a las opciones C y D eliminando la probabilidad 0,66 de ganar $\$ 2.400$ de las dos opciones presentadas en el problema 1. Evidentemente, este cambio produce una reducción mayor en la preferencia cuando se cambia de una opción segura a una con riesgo que cuando tanto el problema original como el reducido son inciertos. Esta situación viola el axioma de independencia sobre el que se basa la teoría de la utilidad esperada.

En una demostración más simple del mismo fenómeno, los autores presentan el siguiente par de problemas.

En el problema 3, los individuos tienen que decidir entre:
A. $(4.000 ; 0,80)$
o
B. (3.000)

En este caso, la mayoría de las personas eligen la opción B.

En el problema 4, los individuos deben decidir entre:
C. $(4.000: 0,20)$
$\mathrm{O}$
D. $(3.000 ; 0,25)$ 
En este caso, la mayoría de las personas eligen la opción C.

En el par de problemas más de la mitad de las personas violaron con sus elecciones los axiomas del comportamiento definidos en la clásica teoría de la utilidad esperada. En las dos situaciones, las personas cambian sus preferencias frente al riesgo. Es decir, en el primer caso, eligen la respuesta segura y, en el segundo caso, eligen la respuesta con riesgo, o viceversa. A este tipo de incumplimiento del axioma, los autores la denominaron "efecto certeza".

En el próximo par de problemas, presentaron cómo los sujetos violan el axioma de sustitución desde un punto de vista meramente probabilístico sin considerar cuestiones monetarias.

En el problema 5, los individuos deben decidir entre:

A. $50 \%$ de probabilidad de ganar un tour de tres semanas por Inglaterra, Francia e Italia.

B. Un tour de una semana por Inglaterra, con certeza.

En este caso, la mayoría de las personas eligen la opción B.

En el problema 6, los individuos deben decidir entre:

C. $5 \%$ de probabilidad de ganar un tour de tres semanas por Inglaterra, Francia e Italia.

D. $10 \%$ de probabilidad de ganar un tour de una semana por Inglaterra.

En este caso, la mayoría de las personas eligen la opción C. Esta es otra demostración de incumplimiento del axioma de sustitución.

Además, Kahneman y Tversky prueban que el "efecto certeza" no es la única causa de incumplimiento del axioma de sustitución. A través de los problemas 7 y 8 , demuestran que este axioma tampoco se cumple a partir de situaciones donde entran en juego la probabilidad y la posibilidad.

En el problema 7 , los individuos deben decidir entre:
A. $(6.000 ; 0,45)$
o
B. $(3.000 ; 0,90)$

En este caso, la mayoría de las personas eligen la opción B.

En el problema 8, los individuos deben elegir entre:
C. $(6.000 ; 0,001)$
o
D. $(3.000 ; 0,002)$ 
En el problema 7, donde las probabilidades de ganar son sustanciales, las personas eligieron a partir de la probabilidad más alta. Sin embargo, en la situación donde ganar es posible pero no probable, como sucede en el problema 8, las personas eligieron en base al monto más alto.

Los problemas recientemente presentados, desde el 1 al 8, demostraron el no cumplimiento de algunos de los axiomas sobre los que se basa la teoría de la utilidad. A raíz de esta situación, los autores comienzan a construir la teoría prospectiva.

Hasta ahora, en los problemas 1 al 8, Kahneman y Tversky analizaron montos positivos; luego continúan su trabajo analizando qué es lo que sucede cuando los montos son negativos. Para ello, utilizan los primeros 4 problemas presentados anteriormente $\mathrm{y}$, únicamente, les cambian el signo a los montos. Encuentran que las decisiones de los sujetos son un mero espejo de lo que responden anteriormente. Es decir, si dentro de los problemas con montos positivos, las personas eligen la opción segura, entonces cuando los montos son negativos, los individuos eligen la opción riesgosa. A este patrón, los autores lo denominan "efecto reflejo".

El problema 9 del trabajo estudió un caso hipotético de seguro probabilístico. Se le plantea a un individuo la siguiente situación: tiene la posibilidad de asegurarse contra los daños que puede sufrir su propiedad, como puede ser un incendio o un robo. Luego de analizar los riesgos y la prima del seguro, el agente no está decidido cuál es su preferencia entre adquirir el seguro o dejar la propiedad sin asegurar. Seguidamente se observa que toma en cuenta que la compañía de seguros ofrece un seguro probabilístico, donde el sujeto paga la mitad de la prima. En el caso de sufrir un accidente, tiene $50 \%$ de probabilidad que la compañía de seguros efectivamente lo cubra del daño percibido y $50 \%$ de probabilidad que la compañía le devuelva las primas pagadas hasta el momento del accidente y no le reconozca nada de las pérdidas. Luego se le preguntaba si, bajo estas circunstancias, estaría dispuesto a comprar el seguro probabilístico. La mayoría de las personas no contrataría el seguro. Esto demuestra que la reducción de la probabilidad de una pérdida a la mitad es mucho menos atractivo que cuando se elimina la probabilidad por completo. Kahneman y Tversky vuelven a hacer una comparación con la teoría de la utilidad 
esperada (con utilidad estrictamente cóncava). De acuerdo con la misma, el seguro probabilístico debería ser preferido al seguro. Es decir, cualquiera sea la posición del individuo a lo largo de $w$ en la figura 5, si él está dispuesto a pagar una prima $y$ de asegurarse de la probabilidad $p$ de sufrir una pérdida $x$, entonces definitivamente debe estar dispuesto a pagar una prima menor $r y$ para reducir la probabilidad de $p$ a $(1-r) p$ de perder $x$, estando $0<r<1$.

Formalmente, si un agente es indiferente entre

$$
\begin{aligned}
& (w-x, p ; w, 1-p)(6) \\
& (w-y)(7)
\end{aligned}
$$

entonces la persona debería preferir el seguro probabilístico

$$
(w-x,(1-r) p ; w-y, r p ; w-r y, 1-p)(8)
$$

por sobre el seguro convencional

$$
(w-y)(9)
$$

De acuerdo con los autores, al momento de decidir entre dos decisiones, las personas tienden a descartar los elementos que estas decisiones comparten y concentrarse en los elementos que las distinguen. En algunos casos, esto lleva a que los individuos sean inconsistentes en sus preferencias. En el trabajo, se define este fenómeno como "efecto aislamiento".

En el problema 10, las personas tienen que considerar un juego de dos etapas. En la primera etapa, los sujetos tienen $75 \%$ de probabilidad de no ganar nada y $25 \%$ de probabilidad de pasar a la siguiente ronda. En la segunda ronda, tienen que elegir entre:
A. $(4.000,0,80)$
o
B. (3.000)

Las personas deben elegir antes de que comience la primera etapa. Nótese que, en los estados finales, la persona tiene $25 \% * 80 \%=20 \%$ de ganar $\$ 4.000$ y $25 \% * 100 \%=25 \%$ de ganar $\$ 3.000$. Entonces, estos estados finales, $(4.000 ; 0,20)$ o $(3.000 ; 0,25)$, son iguales a los del problema 4 . Sin embargo, las preferencias dominantes son diferentes entre los dos problemas. En el problema 10, la mayoría de las personas elige la opción $B$, mientras que en el problema 4 , la mayoría elige la opción $A$. Evidentemente, los sujetos descartan la primera parte del problema y contestan exactamente de la misma manera que lo hicieron en el problema 3. 
En el problema anterior, se ilustra cómo las preferencias pueden ser afectadas a partir de la manera en que las probabilidades son presentadas. En el siguiente par de problemas, se muestra cómo las elecciones pueden ser alteradas si se varían la presentación de los pagos. En el problema 11, se les informa: además de todo lo que ya posee, se le han otorgado \$1.000. Ahora debe elegir entre:
A. $(1.000 ; 0,50)$
o
B. (500)

En el problema 12, se les informa: además de todo lo que ya posee, se le han otorgado $\$ 2.000$. Ahora debe elegir entre:
C. $(-1.000 ; 0,50)$
o
D. $(-500)$

La mayoría de las personas eligen $B$ en el problema 11 y $C$ en el problema 12. Estas preferencias ratifican el mencionado "efecto reflejo" donde las personas son adversas al riesgo en los montos positivos pero amantes al riesgo en los montos negativos. Nótese que en términos de estados finales, las dos situaciones son idénticas. Específicamente:

$$
\mathrm{A}=(2.000 ; 0,50 ; 1.000 ; 0,50)=\mathrm{C} \quad \text { o } \quad \mathrm{B}=(1.500)=\mathrm{D}
$$

El patrón observado en los problemas 11 y 12 es claramente inconsistente con la teoría de la utilidad esperada. En la misma, una utilidad de, imagine, $\$ 100.000$ tiene la misma utilidad si fue obtenida luego de tener $\$ 105.000$ o $\$ 95.000$. Si a esto se le agrega el supuesto que las personas son adversas al riesgo en todo su dominio, la opción segura debería ser siempre preferida a la opción riesgosa. Sin embargo, este par de respuestas muestra que este comportamiento no se ve contemplado por la teoría de la utilidad esperada. El análisis del problema 13 es bastante similar a los anteriormente presentados por lo que no es incluido en este trabajo.

A partir de estas preguntas hipotéticas que demuestran incumplimientos de los axiomas de la teoría de la utilidad esperada y sientan las bases de una nueva función de utilidad, Kahneman y Tversky construyen la teoría prospectiva. Algunos de los elementos sobre los que se basan, ya fueron considerados en intentos de modificar la clásica función de utilidad, a saber: en la función de utilidad propuesta por Markowitz (1952), previamente descripta, también se destaca que la utilidad de las personas está definida por las ganancias y las pérdidas en lugar de riqueza total. Sin embargo, a 
diferencia de Markowitz, Kahneman y Tversky (1979) no proponen una función de utilidad que sea cóncava y convexa tanto en las pérdidas como en las ganancias. Según los autores, esta curva no estaría contemplando el "efecto reflejo".

En conclusión, Kahneman y Tversky proponen una función de valor que tiene tres puntos a destacar:

a. Es definida a partir de desviaciones desde un punto de referencia.

b. En la mayoría de los casos, es cóncava para las ganancias y convexa para las pérdidas.

c. Tiene una pendiente más pronunciada para las pérdidas que para las ganancias.

Esta función de utilidad es graficada en la figura 5 donde se contemplan estas propiedades. En la misma, $w$ es el valor de cada decisión y $U(w)$ es la utilidad que le genera esa decisión. Cabe destacar que esta función de utilidad con forma de $S$ tiene la pendiente más pronunciada en los montos cercanos al punto de referencia mientras que la postulada por Markowitz (1952) es más aplanada en esa zona. Además, a diferencia del trabajo de Markowitz (1952), Kahneman y Tversky determinan que los agentes son, en la mayoría de los casos, adversos al riesgo en todo el dominio de las ganancias y afectos al riesgo en todo el dominio de las pérdidas. Tversky y Kahneman (1992) desarrollan una versión alternativa de la teoría perspectiva. En la misma, consideran las probabilidad acumuladas son transformadas en lugar de las probabilidades individuales. Esto ayuda a solucionar el inconveniente de sobrevalorar las decisiones con baja probabilidad de ocurrencia y subvalorar las decisiones con alta probabilidad de ocurrencia. En esta nueva teoría, los autores determinan que los agentes son adversos al riesgo para las ganancias y afectos al riesgo para las pérdidas que tienen baja probabilidad de ocurrencia; y los individuos son afectos al riesgo para las ganancias y adversos al riesgo para las pérdidas que tienen alta probabilidad de ocurrencia. 
Figura 5. Curva de utilidad con forma de S de Kahneman y Tversky (1979)

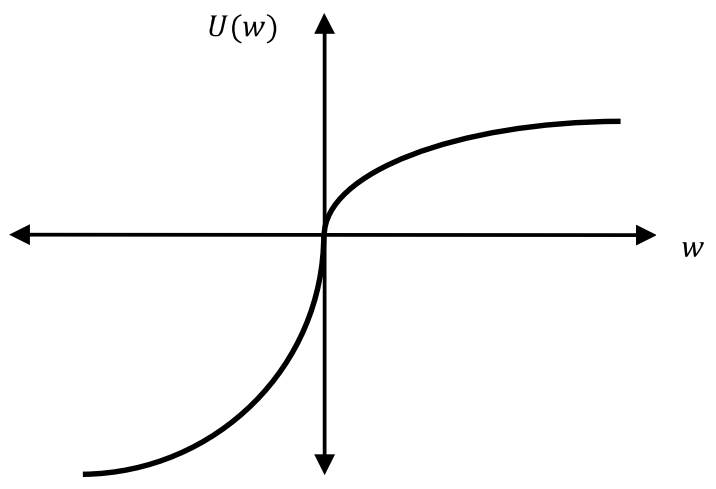

Fuente: elaboración propia.

\section{Cuadro resumen}

En el siguiente cuadro, se exhibe un resumen con los autores y el año que en que propusieron las distintas curvas de utilidad, las características, y la actitud frente al riesgo de los individuos.

Cuadro 1. Características y actitudes de las diferentes curvas de utilidad

\begin{tabular}{lcll}
\hline Autores & Año & Características & Actitud frente al riesgo \\
$\begin{array}{l}\text { Von Neumann y } \\
\text { Morgenstern }\end{array}$ & 1944 & $\begin{array}{l}\text { Considera la riqueza total del } \\
\text { individuo. }\end{array}$ & $\begin{array}{l}\text { Adversos o afecto en todo el } \\
\text { dominio }\end{array}$ \\
\hline Friedman y Savage & 1948 & $\begin{array}{l}\text { Considera el ingreso del } \\
\text { individuo. }\end{array}$ & $\begin{array}{l}\text { Adverso en niveles de ingresos } \\
\text { bajos y altos; afecto en la } \\
\text { transición de los mismos. }\end{array}$ \\
\hline Markowitz & 1952 & $\begin{array}{l}\text { Propone un punto de referencia; } \\
\text { analiza diferentes valores } \\
\text { monetarios; los agentes evitan } \\
\text { apuestas justas. }\end{array}$ & $\begin{array}{l}\text { Ganancias bajas: afecto. } \\
\text { Ganancias altas: adverso. } \\
\text { Pérdidas bajas: adverso. } \\
\text { Pahneman y Tversky }\end{array}$ \\
\hline \multirow{2}{*}{1979} & $\begin{array}{l}\text { Se crea a partir de un punto de altas: afecto. } \\
\text { referencia; los agentes evitan } \\
\text { apuestas justas; analiza un solo } \\
\text { valor monetario. }\end{array}$ & $\begin{array}{l}\text { Adverso frente a las ganancias } \\
\text { yecto frente a las pérdidas. }\end{array}$ \\
\hline
\end{tabular}

Fuente: elaboración propia. 


\section{Conclusiones}

Se resumieron los principales modelos, sus características y las actitudes frente al riesgo de los agentes. Todas presentan un común denominador, un ser humano promedio que prefiere mayor a menor satisfacción. No obstante, se diferencian respecto de la reacción que este tiene frente a potenciales incremento de ganancias o pérdidas, puntos de referencia y estados de riqueza. La clásica formulación de Von Neumann y Morgenstern (1944) trabaja con riqueza total (acumulaciones) siendo afectos o adversos en todos los dominios de la función de utilidad. A partir de aquí, surgen curvas en forma de " $S$ ". Friedman y Savage (1948) consideran los ingresos de las personas y comportamientos variables según la persona se encuentre en segmentos extremos o intermedios de la curva. Markowitz (1952) para las ganancias altas y pérdidas bajas concibe un ser adverso y afecto en los tramos opuestos, además de incorporar puntos de inflexión. Cabe destacar que Markowitz introduce el criterio media-varianza como herramienta para aproximar, vía precios observados, el criterio de maximización de utilidad. Finalmente, Kahneman y Tversky (1979) plantean un nuevo paradigma a través de la teoría de las perspectivas o prospectiva, siendo este cuerpo teórico la piedra basal de las finanzas conductuales (Behavioral Finance), donde los modelos financieros producto de las finanzas neoclásicas son atravesados por sesgos, respecto de la decisión racional a partir de heurísticas activadas en la toma de decisiones riesgosas de los agentes. 


\section{Referencias}

Allais, M. (1988). An Outline of My Main Contributions to Economic Science. Economic Sciences, 233-252.

Allais, M. (1953). Le Comportement de l'Homme Rationnel devant le Risque: Critique des Postulats et Axiomes de l'Ecole Americaine. Econometrica, 21 (4), 503-546.

Bernoulli, D. (1738). Publicado en 1954. Exposition of a New Theory on the Measurement of Risk. Econometrica, 22 (1), 23-36.

Chaz Sardi, M. C. (2015). Garantías: Problemas de Información y Errores de Percepción. La Visión Tradicional y el Aporte de la Economía Conductual. Tesis Doctoral en Economía. Universidad Nacional del Sur. Disponible en Internet: http://repositoriodigital.uns.edu.ar/bitstream/ 123456789/2357/1/Tesis\%20DOCTORAL\%20Chaz\%20Sardi.pdf

Dehling, H. G. (1997). Daniel Bernoulli and the St. Petersburg Paradox. Nieuw Archief Voor Wiskunde, 15 (3), 223-227.

Ederington, L. (1996). Mean-variance as an approximation to expected utility maximization: semi ex-ante results. In: Advences in Financial Economics Vol. 1. W. M. Mark Hirschey, Ed.

Friedman, M., \& Savage, L. J. (1948). The Utility Analysis of Choices Involving Risk. The Journal of Political Eocnomy , 56 (4), 279-304.

Huygens, C. (1657). Publicado en 1980. De ratiociniis in ludo aleae. Ex officina J. Elsevirii.

Kahneman, D. \& Tversky, A. (1979). Prospect Theory: An Analysis of Decision Under Risk. Econometrica, 47 (2), 263-292.

Kahneman, D. \& Tversky, A. (1979). Publicado en 1987. Teoría Prospectiva: Un Análisis de la Decisión Bajo Riesgo. Infancia y Aprendizaje, (30), 95-124.

Levy, H. \& Markowitz, H. (1979). Apporximating expected utility by a function of mean and variance. American Economic Review, 69 (3), 308-317.

Markowitz, H. (2014). Mean-variance approximations to expected utility. European Journal of Operational Research (234), 346-355.

Markowitz, H. (1952). Portfolio Selection. Journal of Finance, 7 (1), 77-91.

Markowitz, H. (1959). Portfolio Selection: Efficient Diversification of Investments. 2 ed. Wiley Yale Universitiy Press. 
Markowitz, H. (1991). Foundations of Portfolio Theory. Journal of Finance, 46 (2), 469-477.

Markowitz, H. (1952). The Utility of Wealth. Journal of Political Economy, 60 (2), 151-158.

Savage, L. J. (1954). The Foundations of Statistics. Nueva York: John Wiley \& Sons.

Simaan, Y. (1993). What is the opportunity cost of mean-variance investment strategies? Management Science, 39 (5), 578-587.

Simon, H. A. (1955). A Behavioral Model of Rational Choice. The Quarterly Journal of Economics, 69 (1), 99-118.

Simon, H. A. (1975). The Functional Equivalence of Problem Solving Skills. Cognitive Psychology, 7, 268-288.

Simon, H. A. (1978). Rationality as Process and as Product of Thought. The American Economic Review, 68(2), 1-16.

Simon, H. A. (1979). Rational Decision Making in Business Organizations. The American Economic Review, 69 (4), 493-513.

Simon, H. A. (1986). The Information Processing Explanation of Gestalt Phenomena. Computers in Human Behavior, 2, 241-255.

Tversky, A. \& Kahneman, D. (1992). Advances in Prospect Theory: Cumulative Representation of Uncertainty. Journal of Risk and Uncertainty, 5:297-323.

Von Neumann, J., \& Morgenstern, O. (1944). Theory of Games and Economic Behavior. Princeton, New Jersey: Princeton University Press. 
Evolución de las funciones de utilidad para la toma de decisiones

1 Además de todas las ramificaciones que tiene el concepto, en particular, en lo que respecta a la valuación de activos financieros y reales.

2 Bernoulli sostiene que no hay dudas que la ganancia de 1.000 ducados va a ser más significativa para un hombre pobre que para un hombre rico, aunque en términos absolutos ambos perciban la misma cantidad de dinero. (Bernoulli, 1738).

3 Por lo tanto, se supone un individuo adverso al riesgo, con forma logarítmica, siendo la segunda derivada de la función de utilidad negativa.

4 El gráfico que representa al agente adverso al riesgo es introducido primeramente por Bernoulli (1738).

5 Para un sujeto neutral al riesgo $U^{-}{ }^{-}{ }_{x}=0$ ya que $U(\$ 1)=0,5 \times U(\$ 2)+0,5 \times U(0)$; reordenando y multiplicando por 2 ambos lados; $U(\$ 1)-U(\$ 0)=U(\$ 2)-U(0)$. En el caso de una persona afecta al riesgo $U{ }^{-}{ }_{x}>0$ donde $U(\$ 1)<0,5 \times U(\$ 2)+0,5 \times U(0)$; reordenando y multiplicando por 2 ambos lados; $U(\$ 1)-U(\$ 0)<U(\$ 2)-U(0)$.

${ }^{6}$ La intención, tanto de este como de los siguientes gráficos, no es determinar el nivel de preferencia al riesgo sino dejar en claro únicamente su preferencia al riesgo mediante la concavidad o convexidad de las curvas.

7 Una apuesta justa es cuando las diferentes alternativas posibles tienen el mismo valor esperado.

8 Más adelante, se explicará la manera en la que el autor construye una nueva curva de utilidad.

9 Por ejemplo, rendimientos logarítmicos de los activos, siendo $\ln$ el logaritmo natural y $r$ el rendimiento. 